\title{
Editorial
}

\section{Semiconductor Nanomaterials for Energy Conversion and Storage}

\author{
Xiang Wu, ${ }^{1}$ Xijin Xu, ${ }^{2}$ Chuanfei Guo, ${ }^{3}$ and Chee Kiang Ivan Tan ${ }^{4}$ \\ ${ }^{1}$ College of Chemistry and Chemical Engineering, Harbin Normal University, Harbin 150025, China \\ ${ }^{2}$ School of Physics and Technology, University of Jinan, Jinan 250022, China \\ ${ }^{3}$ Department of Physics, University of Houston, Houston, TX 77004, USA \\ ${ }^{4}$ Institute of Materials Research and Engineering (IMRE), A STAR, Singapore 117602
}

Correspondence should be addressed to Xiang Wu; wuxiang05@163.com

Received 14 December 2014; Accepted 14 December 2014

Copyright (C) 2015 Xiang Wu et al. This is an open access article distributed under the Creative Commons Attribution License, which permits unrestricted use, distribution, and reproduction in any medium, provided the original work is properly cited.

Energy and environmental problems have raised great concerns in recent decades. On one hand, people have an increasing demand on energy for which the consumption causes many environmental problems. On the other hand, clean energy is significantly increasing, but the consumption of fossil energy is still abundant. To date, beside conventional energy including fossil energy, hydroelectric power, and wind energy, scientists have developed several technologies to generate energy by converting solar energy into electricity or heat, by converting heat energy to electricity, or by converting mechanical energy to electricity. Indeed, generation of energy is only one of many issues for energy engineering. Now we have three big challenges in the area of new energy: generation, transport, and storage of energy. A demonstration that energy can be generated, transported, and stored by applying new materials and nanostructures is of great importance in both scientific research and practical applications.

As Guest Editors, we have successfully organized two special issues titled Self-Assembly of 1D Semiconductor Nanostructures and Metal Oxide Heterostructures for Water Purification in the past two years. Now it is our pleasure to finish the third special issue which is presented herein. This special issue covers all topics related to semiconductor materials and nanostructures for the generation, transport, and storage of energy, such as solar cells, supercapacitors, and lithium ion batteries. This special issue consists of one review paper and ten research papers that are contributed from Chinese scientists.
The review article by $\mathrm{Xu}$ et al. summarized the syntheses of phase-pure $\mathrm{SnO}_{2}$ hierarchical structures with different morphologies such as nanorods, nanosheets, and nanospheres, as well as their modifications by doping and compositing with other materials. They reviewed the design of $\mathrm{SnO}_{2}$-based nanostructures with improved performance in the areas of lithium-ion batteries and supercapacitors. The article by $\mathrm{X}$. Ji et al. reported the growth of carbon-coated $\mathrm{Zn}_{2} \mathrm{SnO}_{4}$ materials by a facile hydrothermal method using the $\mathrm{Zn}_{2} \mathrm{SnO}_{4}$ octahedron as the precursor and glucose as carbon source and studied their electrochemical properties, revealing that carbon-coated $\mathrm{Zn}_{2} \mathrm{SnO}_{4}$ exhibits high rate capability and long cycle life compared with bare $\mathrm{Zn}_{2} \mathrm{SnO}_{4}$, indicating its promising application as anode for lithiumion batteries. Q. Sun and his coworkers reported a portable cross-linked $\mathrm{ZnO}$ nanowalls/bamboo composites catalyst. Compared with those powder photocatalysts, the as-prepared photocatalyst was very easily separated from the dye aqueous solution and cyclically used.

The work from X. Wu studied the optical and magnetic properties of diluted magnetic semiconductors $\mathrm{Zn}_{1-x} \mathrm{Fe}_{x} \mathrm{O}$ nanoparticles with different doping consistency. Photoluminescence spectra exhibited a slight blue shift and the UV emission is annihilated with the increase of $\mathrm{Fe}^{3+}$ concentration. The obvious ferromagnetic properties are found for all doped samples at room temperature, and the saturation magnetization is enhanced with the increase of iron doping content. X. Ji et al. designed a new $\mathrm{CuO}$ film on Ti substrate 
$(\mathrm{CuO} / \mathrm{Ti})$ electrode by effectively taking advantage of the conductivity and biocompatibility of the substrate. Then they investigated the application of this new electrode for glucose determination, demonstrating that the fabricated glucose biosensor exhibited a high sensitivity, fast response, an appropriate linear range, and good stability. The good analytical performance, low cost, and one-step preparation method make this electrode material promising for the development of a nonenzymatic glucose sensor. Wang et al. studied the nanorod based LEDs, and the light output of dipole power in the planar LED, the nanorod LED, and the LSP assisted LED were also studied by two-dimensional finite difference time domain method.

P. Yan et al. reported the synthesis of hierarchical $\mathrm{CoO}$ nano/microstructures via a hydrothermal method followed by a subsequent thermal annealing process. They studied electrochemical properties of as-synthesized hierarchical $\mathrm{CoO}$ nano/microstructures, revealing a high initial capacity of about $1370 \mathrm{mAh} / \mathrm{g}$ and superior cycle stability. Good rate capability is also obtained in $\mathrm{CoO}$ nano/microstructures as anode materials for LIBs.

The paper by Wang studied the electronic structures and optical properties of $\mathrm{Sn}_{15} \mathrm{FeO}_{32}$ with electron-injection by means of the full-potential linearized augmented plane-wave method (FP-LAPW). Their results showed that Fe-doped $\mathrm{SnO}_{2}$ materials are all direct transition semiconductors. The Fermi level goes into conduction band gradually and the band gap decreases with the increase of electron injection. The peaks of optical properties, such as the imaginary part of dielectric function and absorption spectra, change greatly at low energy. The absorption spectra exhibit blue shift, and the optical absorption edge increases, which are consistent with the change of the band gaps. C. Jin et al. reported the bamboo with multifunction of superhydrophobicity, UVresistance, and fire-resistance fabricated by coating with $\mathrm{ZnO}$ nanosheet networks via a hydrothermal method and subsequent modification with FAS-17.

$\mathrm{Li}$ and Lu's work studied in situ preparation of anatase titania $\left(\mathrm{TiO}_{2}\right)$ homogeneously dispersed in cellulose aerogels substrates. The obtained anatase titania/cellulose (ATC) aerogels showed interconnected three-dimensional architecture surrounded by numerous anatase $\mathrm{TiO}_{2}$ particles aggregates. Compared with commercially available Degussa $\mathrm{P}_{25}$, ATC aerogels displayed more excellent photocatalytic activities for Rhodamine $\mathrm{B}$ and methyl orange degradations under UV radiation. Finally, Yong et al. synthesized novel graphitic carbon nitride/ $\mathrm{KTaO}_{3}\left(\mathrm{~g}-\mathrm{C}_{3} \mathrm{~N}_{4} / \mathrm{KTaO}_{3}\right)$ nanocomposite photocatalysts by a facile and simple ultrasonic dispersion method. Compared to either $\mathrm{g}-\mathrm{C}_{3} \mathrm{~N}_{4}$ or $\mathrm{KTaO}_{3}$, the composite photocatalysts show significantly increased photocatalytic activity for degradation of Rhodamine $\mathrm{B}(\mathrm{RhB})$ under visible light irradiation. They thought the increased photocatalytic performance of the composite could be attributed to the enhanced photogenerated charge carrier separation capacity.

We sincerely hope that the special issue can provide a valuable reference to the current development and future research of energy storage and conversion. Finally, we also hope that the collection of these articles will inspire researchers and stimulate new ideas in relative research field.

\section{Acknowledgment}

As Guest Editors, we would like to give many thanks to all of the above authors for their contributions to this special issue. At the same time, we are also grateful to the selfless referees for their hard work in reviewing these manuscripts.

Xiang $W u$ Xijin $X u$

Chuanfei Guo

Chee Kiang Ivan Tan 

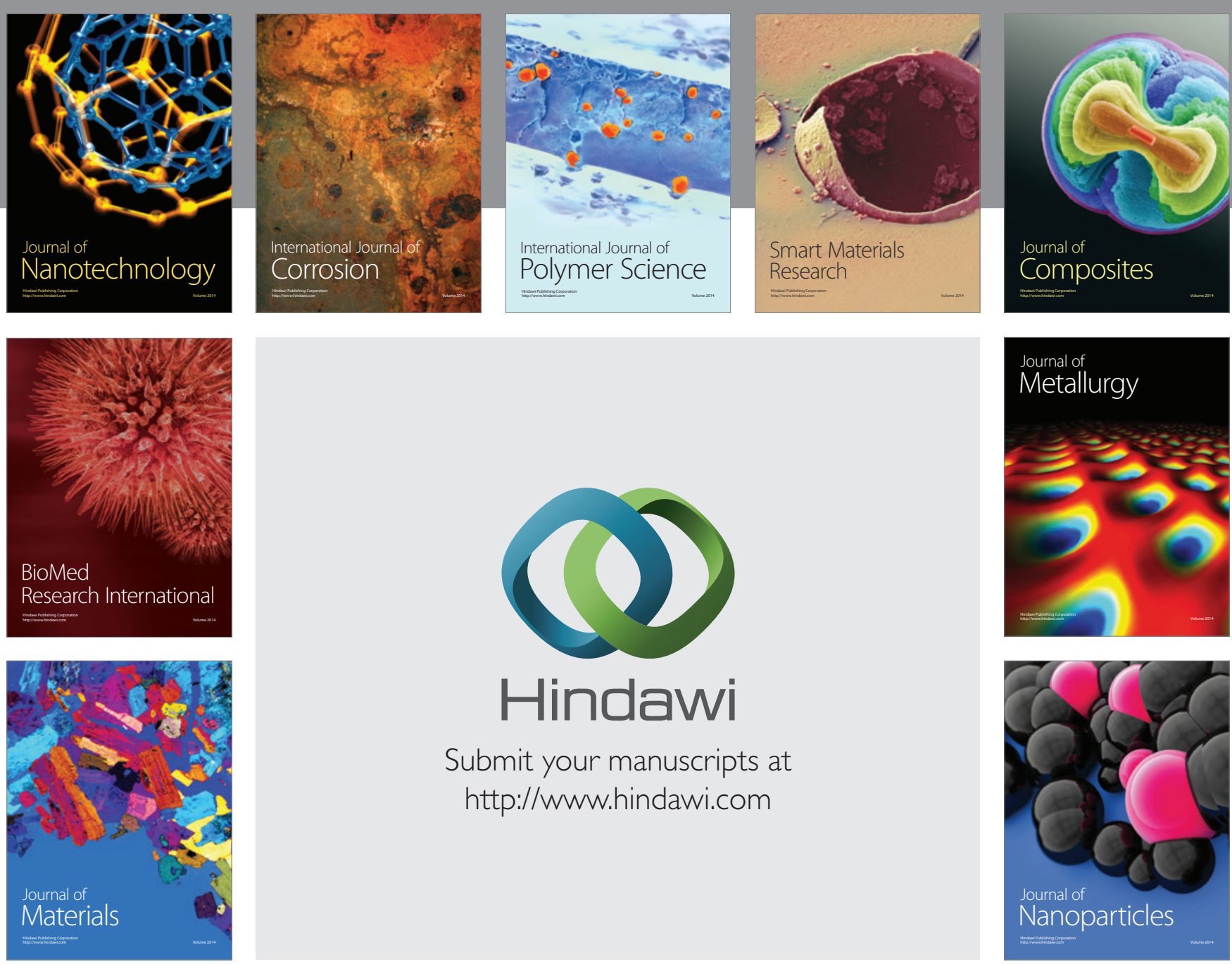

Submit your manuscripts at http://www.hindawi.com
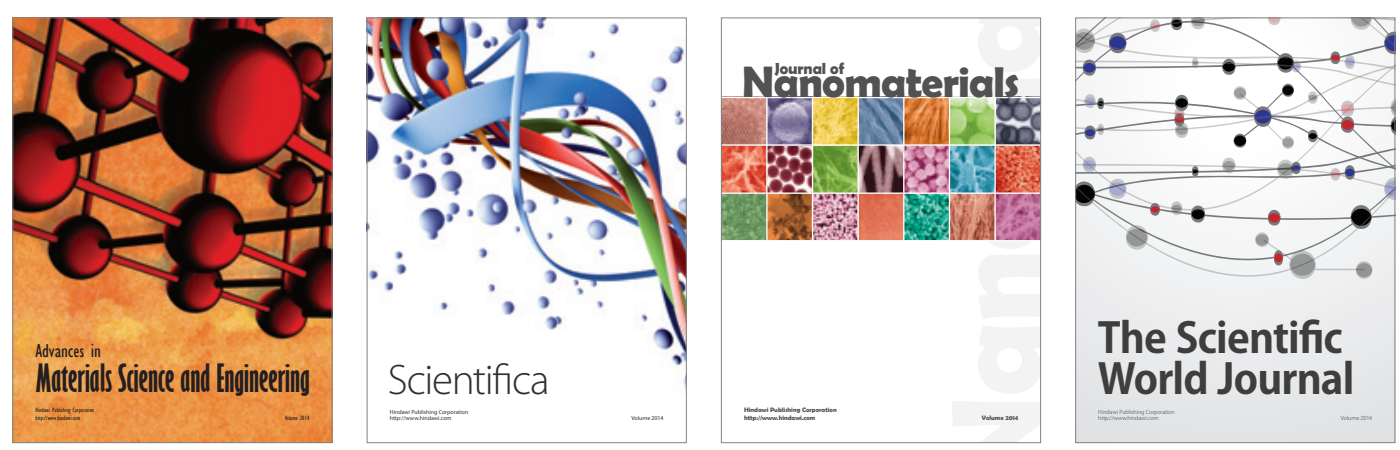

\section{The Scientific World Journal}
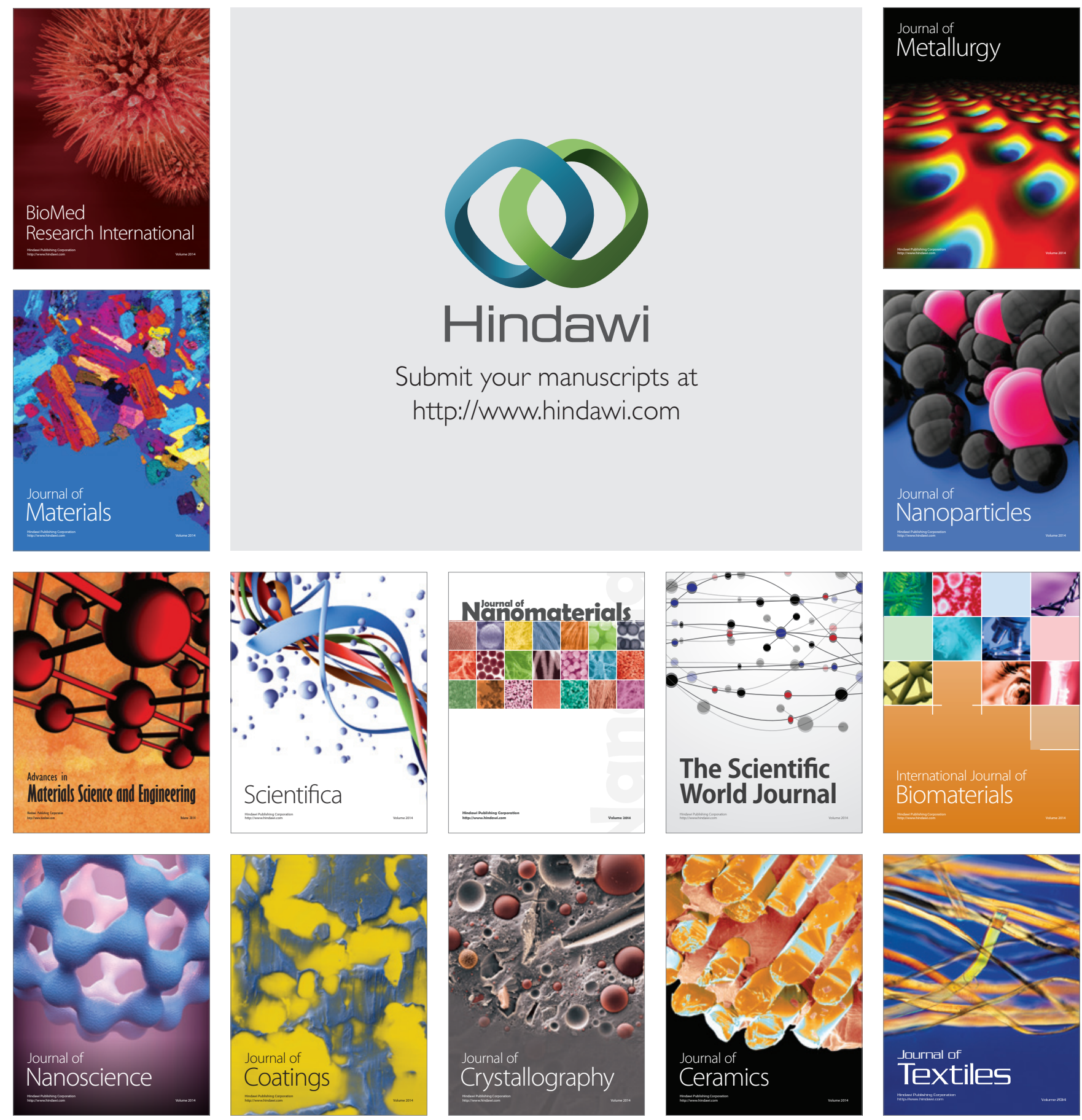